\title{
CONSIDERACIONES SOBRE UN NUEVO Y NOTABLE PUÑAL CALCOLÍTICO ATRIBUIBLE AL GRUPO TREILLES DEL MUSEU D’ARQUEOLOGÍA DE CATALUNYA EN BARCELONA
}

\author{
POR
}

\author{
JORDI ROVIRA I PORT
}

\section{RESUMEN - ABSTRACT}

Aunque ignoramos su procedencia concreta, el Museu d'Arqueologia de Barcelona conserva en sus colecciones un magnífico ejemplar de puñal de cobre tipológicamente atribuible a las series de puñales denominados genéricamente como «à encoche bilatérale» y «à soie courte crantée». Se trata de piezas asimilables mayoritariamente a la metalúrgia precampaniforme del Grupo «des Treilles», aunque ciertos ejemplares aparecen también en los grupos culturales prehistóricos franceses de Fontbuisse y Véraza.

En el artículo se efectúan apreciaciones sobre su tipologia, la filiación y la datación del puñal, así como sobre su probable composición metalográfica.

Althrough we were unaware of its concret origin, the Museu d'Arqueologia of Barcelona preserves in his collections a magnificent example of copper dagger assimilable to the series of daggers so-called «à encoche bilatérale» and «à soie courte crantée». These are pieces assimilated principally to the metallurgy of the pre-bell beakers of the Treilles Group, althrough certain pieces appear also in the prehistoric cultural groupes of Fontbuisse and Véraza.

We present suggestions about its typology, filiation and the chronology of this dagger, as well as about its likely metallic composition.

\section{PALABRAS CLAVE - KEY WORDS}

Puñal, Cobre, Metalúrgia precampaniforme, Treilles, Fontbuisse, Véraza.

Dagger, Copper, Pre-bell beaker metallurgy, Treilles, Fontbuisse, Véraza.

\section{UNAS PRECISIONES INTRODUCTORIAS}

El Museu d'Arqueologia en Barcelona conserva entre sus colecciones un magnífico ejemplar de puñal metálico el cual, desde una fecha ignorada, permanecia almacenado junto con otros muchos elementos metálicos de filiaciones y procedencias diversas, aunque, en su inmensa mayoría, atribuibles tipológicamente a los dos siglos anteriores al cambio de Era y a etapas alto- imperiales romanas. La pieza en cuestión no se hallaba inventariada ni portaba fecha ni inscripción alguna sobre su superficie, y, por tanto, carecemos de cualquier indicación sobre su procedencia, hallazgo e ingreso en aquella institución. En este sentido, caben pocas opciones: o ingresó en el museo barcelonés procedente directamente de algun yacimiento arqueológico peninsular, — quizás del nordeste peninsular - cosa poco probable 
aunque no imposible, o llegó a esta institución junto con otros materiales a consecuencia de alguna adquisición o donación procedente de la zona gala del Aveyron. O, simplemente, se trata de una pieza ingresada mediante algún acuerdo de intercambio de objetos arqueológicos, práctica harto frecuente a lo largo de la primera mitad del siglo XX. Así las cosas, y a la espera de que alguna otra documentación pueda proporcionar información sobre estas cuestiones ahora citadas, hemos creido oportuno el dar a conocer este muy interesante elemento metálico el cual, aunque descontextualizado, nos ofrece la posibilidad de volver de nuevo sobre los aún insuficientemente estudiados y difundidos fondos arqueológicos del museo barcelonés.

\section{EL PUÑAL}

La pieza, desde un punto de vista estrictamente tipológico, consiste en un puñal de morfologia largamente foliácea caracterizado en su zona proximal por una notable y bien diferenciada lengüeta triangular/ojival y sendas escotaduras laterales de separación entre la zona de enmangue y la hoja de la pieza. La zona distal se encuentra formada por una hoja de cortes o laterales convexos - especialmente a partir de la zona medial- que convergen en una punta o extremo distal aguzado. (Fig. 1).

La lengüeta, por su parte, y a lo largo de su perímetro lateral presenta algunas pequeñas escotaduras habilitadas para facilitar el enmangue. Señalaremos también la existencia en cada una de las caras del puñal de una doble y casi imperceptible nervadura que discurre a lo largo de su eje longitudinal desde, aproximadamente, la zona medial de la lengüeta, hasta la punta distal de la pieza. La sección transversal de la hoja es suavemente biconvexa finalizando lateralmente en sendos cortes bien afilados. El acabado del objeto implicó una notable labor de martillado y pulimento. En cuanto a su conservación, diremos que es buena a pesar de que la pieza fue ligeramente doblada de antiguo en su tercio distal y muestra abundantes muescas en los filos, algunos golpes, rayados y excamaciones, así como un número notable de cloruros a lo largo de toda la superficie. Esta última, por su lado, ha llegado hasta nosotros rugosa e irregular, sobre todo por lo que respecta a la lengüeta y al tercio distal, mostrando una gama de colores y tonalidades predominantes que van desde el marrón oscuro y el granate hasta los verdes oscuros, el verde esmeralda y el beig.

Las dimensiones de la pieza son las siguientes: Longitud máxima: $204 \mathrm{~mm}$; longitud de la lengüeta: $44 \mathrm{~mm}$; anchura máxima: $34 \mathrm{~mm}$, y grosor máximo: $4 \mathrm{~mm}$. En cuanto al peso: 82 gramos.

En último lugar, y por lo que respecta al número de registro correspondiente al inventario general del museo que le ha sido asignado recientemente, éste es el siguiente: 34.067.

\section{FILIACION, DATACION Y COMENTARIOS}

A pesar de su descontextualización, el puñal que nos ocupa es un notable exponente de la panoplia metálica elaborada en cobre dentro del marco geográfico de las Grands Causses, en el departamento francés de L'Aveyron, y en el seno de comunidades transicionales que ilustran el paso desde un neolitico medio/reciente de filiación Chassey hasta el Calcolítico inicial precampaniforme. (Clottes y Costantini, 1976: 286-287). De hecho, nuestra pieza debe ser asociada al numeroso grupo de puñales denominados genéricamente como «à encoche bilatérale» y «à soie courte crantée» (Briard y Mohen, 1983: 17-18) y considerados de manera global como paradigmáticos ejemplos de una notable metalurgia local del cobre (3500/3000- 
2200 a.C.) en el seno de poblaciones del neolitico final precampaniforme asimiladas al denominado Grupo «des Treilles». (Costantini, 1980: 233-239). (Fig. 2).

Puñales paralelizables con este ejemplar de Barcelona aparecen también en contextos de cultura material de los grupos Fontbuisse y Véraza en unas fechas que oscilarian entre el 2500 y el 2000 a,C. en tanto que su repartición geográfica coincide grosso modo con los actuales territorios departamentales del Aveyron y La Lozère. Así, el puñal del Museo de Barcelona seria un elemento metálico más a añadir a la ya larga lista de más de un centenar de puñales Calcolíticos-Bronce antiguo de distintas tipologias aparecidos a lo largo y ancho de estos departamentos del mediodia francés — de los cuales más de cincuenta han aparecido en la zona de las Grands Causses-, aunque con la particularidad reseñable de sus dimensiones. (Gasco, 1980: 397-415). En efecto, los puñales que caracterizan entre otros elementos a la primera metalúrgia local del Aveyron, la Lozère y El Gard, muestran en sus ejemplares más notables clasificables concretamente en el grupo de "poignards à courte soie crantée» unas longitudes de hoja que oscilan entre los 100 y los $180 \mathrm{~mm}$., alcanzando los $122 \mathrm{~mm}$. el puñal exhumado en la Grotte des Cascades y llegando hasta los 190 mm. reseñados en 1980 por Costantini para alguna pieza determinada. Así las cosas, el puñal de Barcelona que alcanza los 204 mm., sería el de mayor longitud de los exhumados hasta ahora o, por lo menos, se hallaria, sin duda, entre las piezas de mayores dimensiones del amplio muestrario atribuible a esta producción local de puñales ya desde etapas precampaniformes.

Por otro lado, hay que apuntar que otros ejemplares de puñales paralelizables con el elemento que nos ocupa proceden tanto del conjunto de puñales exhumados en el túmulo $\mathrm{X}$ de Freyssinel (Lozère) como de otros muchos yacimientos del Midi, caso del sepulcro megalítico de Baldare (Saint-Léons, Aveyron), o de las cavidades bautizadas como Grotte des Gaches (Veyreau, Aveyron) y Grotte I des Cascades (Creissels, Aveyron), esta última con un ejemplar de puñal que muestra una doble serie lateral de cuatro escotaduras. (Morel, 1934: 177-194; Costantini, 1965: 649-664).

Para finalizar, no deja de ser problemática tanto la localización del foco metalúrgico que originó la pieza como la procedencia de las mineralizaciones utilizadas en su composición, aunque cabe recordar que la tipologia de la pieza barcelonesa encaja bien con algunas de las distintas variantes de esta familia de puñales fruto de las producciones metalúrgicas antes citadas. En este sentido, si un futuro análisis espectrométrico revela que su contenido en impureza de plomo es prácticamente nulo, o igual o inferior a 0’005, es muy posible que se confirme esta primera apreciación de hallarnos ante un típico producto metálico elaborado a partir de mineral cúprico extraido de los yacimientos naturales de la zona de Cabrières, (Ambert et alii, 1985: 91-95; Esperou, 1992: 26-31) aunque sin olvidar los matices futuros que puedan hacerse a esta posibilidad derivados de las nuevas perspectivas abiertas por los últimos análisis sobre muestras de carbonatos de cobre con ínfimos porcentajes de impurezas de plomo procedentes de la zona de las Cévennes. (Ambert, 1992: 51-58; Costantini, 1992: 59-66).

JORDI ROVIRA I PORT

Museu d'Arqueologia de Catalunya.Barcelona. 


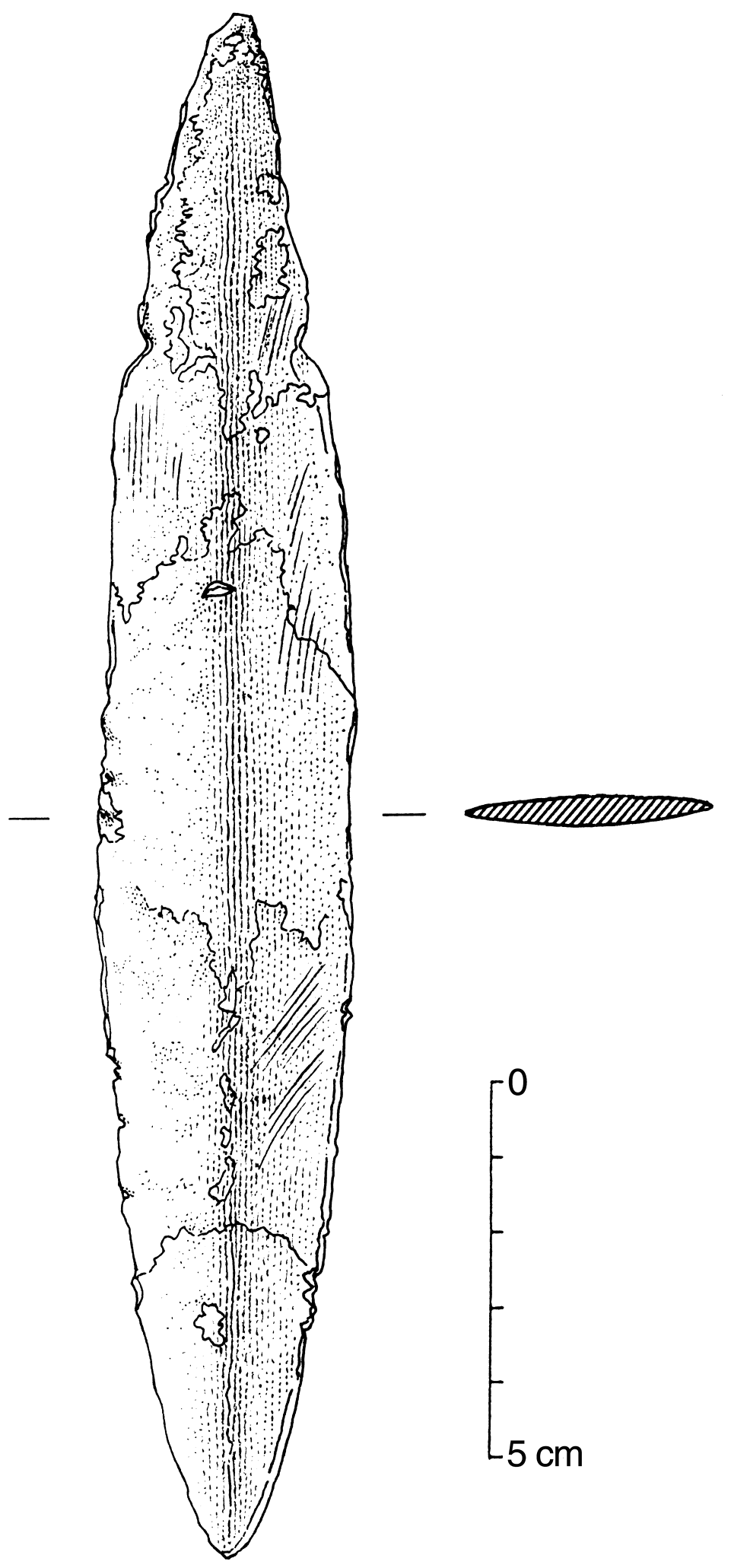

Fig. 1.- Puñal calcolítico atribuible al grupo «des Treilles». 


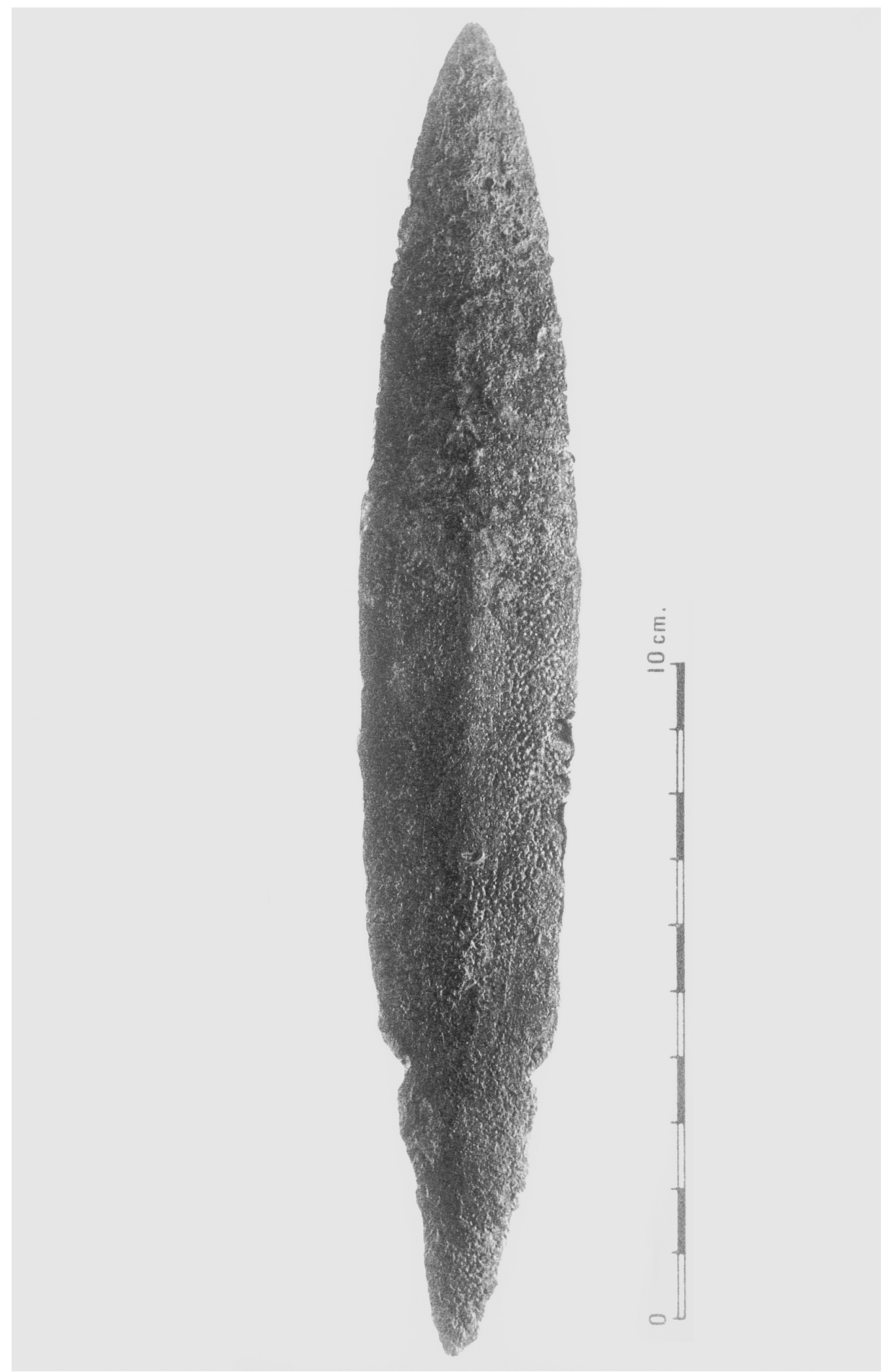

Fig. 2.- Puñal «à encoche bilatérale». Es apreciable una muy ligera cresta longitudinal que alcanza desde el extremo proximal hasta el distal. 


\section{BIBLIOGRAFIA}

AMBERT, P.; BARge, H.; Bourhis, J.R.; EsPerou, J.L. (1985): «Mise en evidence, âge et niveau technique des explotations préhistoriques cuprifères de Cabrières (Hérault).» Archéologie en Languedoc, 4: 91-95.

AMBERT, P. (1992): «L'émergence de la métallurgie Chalcolithique dans le Midi de la France.» Le Chalcolithique en Languedoc. Ses relations extra-regionales. Archéologie en Languedoc 1990-1991. Colloque International Hommage au Dr. Jean Arnal. Saint-Mathieu-de-Tréviers, 1990, Lattes: 51-58.

BRIARD, J; MoHen, J.P. (1983): Typologie des objets de l'Age du Bronze en France. Fascicule II: Poignards, Hallebardes, Pointes de lance, Pointes de flèche, Armement défensif. Société Préhistorique Française. Commission du Bronze. Paris: 17-18.

Clottes, J.; Costantini, G. (1976): «Les civilisations néolithiques dans les Causses.»La Préhistoire Française II. Les civilisations néolithiques et protohistoriques de la France. (J. Guilaine, dir.). IX Congrés UISPP. Nice, 1976, Paris: 286-287.

Costantini, G. (1965): «La grotte I des Cascades. Commune de Creissels (Aveyron).» Bulletin de la Société Préhistorique Française, LXII: 649-664.

Costantini, G. (1980): «La métallurgie du Groupe des Treilles». Colloque. Le Groupe de Véraza et la fin des temps néolithiques dans le Sud de la France et la Catalogne (J. Guilaine, dir.). CNRS. Paris: 233-239.

CostantinI, G. (1992): «Les productions métalliques du groupe des Treilles et leur répartition dans le Midi de la France.» Le Chalcolithique en Languedoc. Ses relations extra-regionales. Archéologie en Languedoc 1990-1991. Colloque International Hommage au Dr. Jean Arnal. Saint-Mathieude-Treviers, 1990, Lattes: 59-66.

ESPEROU, J.L. (1992): «Cabrières, et les Cuivres à Sb/Ag du Sud de la France.» Bulletin de la Société Préhistorique Française, 89/1: 26-31.

Gasco, J. (1980): «Les Poignards en cuivre du Midi bas-languedocien». Bulletin de la Société Préhistorique Française, 77/10-12: 397-415.

Morel, CH. (1934): «Le Tumulus $n^{\circ} \mathrm{X}$ du Freyssinel (Causse de Sauveterre-Lozère)», Bulletin de la Société Préhistorique Française, XXXI: 177-194. 